\title{
Application of chiral sulfoxides in asymmetric synthesis
}

\begin{abstract}
Chiral sulfoxides are used as a toolbox for the synthesis of enantiomeric/diastereomeric compounds, which are used as precursors for the pharmaceutically/chemically important molecules. The current review focuses on applying these chiral sulfoxides towards the synthesis of the compounds having stereogenic center. In general, the stereogenic center induced by the sulfoxide is able to direct the stereochemistry of further transformation necessary to complete the total synthesis of bioactive molecules. The nature of the reactive conformation of the sulfoxide is strongly dependent on the nature of the substituents at $\mathrm{C}-\alpha$ and/or $\mathrm{C}-\beta$.
\end{abstract}

Volume 2 Issue 2 - 2018

\author{
Ganapathy Subramanian Sankaran,' \\ Srinivasan Arumugan, ${ }^{2}$ Sivaraman \\ Balasubramaniam ${ }^{3}$ \\ 'University of Massachusetts Medical School, USA \\ ${ }^{2}$ Department of Science and Humanity (Chemistry), Karunya \\ Institute of Technology and Sciences, India \\ ${ }^{3}$ Indian Institute of Technology Madras, Chennai, India
}

\author{
Correspondence: Sivaraman Balasubramaniam, Senior \\ Research Scientist, Indian Institute of Technology Madras, \\ Chennai, India, Tel +9177 I880 51।3, Email \\ sivaramaniitm@gmail.com
}

Received: March 07, 2018 | Published: March 29, 2018

\section{Introduction}

Over the last three decades, the sulfinyl group has received considerable attention in asymmetric synthesis ${ }^{1-5}$ as a chiral tool. The sulfinyl group is widely used as to bring about numerous asymmetric transformations. The effectiveness of the sulfoxide in diastereoselective auxiliary-induced reactions is mainly due to the steric and stereo electronic differences existing between the substituents on the stereogenic sulfur atom: a lone electron pair, oxygen, and two different carbon ligands, which are able to differentiate the diastereotopic faces of a proximal or even remote reaction center. Besides the high configurational stability of the sulfinyl group, ${ }^{6,7}$ the existence of several efficient methods to obtain homochiral sulfoxide as well as their synthetic versatility has led to a substantial growth of the use of these chiral starting materials in the synthesis of enantiomerically enriched compounds and in the total synthesis of numerous biologically active natural products.

\section{An overview on the synthesis of homochiral sulfoxides}

\section{Asymmetric oxidation of the thioethers}

A variety of methods are available to obtain optically active sulfoxides, these include optical resolution, asymmetric oxidation and asymmetric synthesis. The first example of asymmetric oxidation of sulfides to sulfoxides were independently reported by Pitchen et al. ${ }^{8}$ and Di Furia et al. ${ }^{9}$ using a modified Sharp less epoxidation reagent $\left[\mathrm{Ti}\left(\mathrm{O}^{\mathrm{P}} \mathrm{Pr}\right)_{4} /(+)-\mathrm{DET} /{ }^{\mathrm{t}} \mathrm{BuOOH}\right]$. Further development of this methedology $y^{10,11}$ showed an increase in the optical purity of the resulting sulfoxide by replacing the tert-butylhydroperoxide with cumenehydroperoxide.

The use of (R)-(+)-binaphthol ${ }^{12}$ instead of DET (Figure 1) as chiral ligand improved the modest ee $(60-70 \%)$ achieved in the transformation of some methyl aryl sulfides into sulfoxides to $96 \%$ ee by taking advantage of the kinetic resolution process which occurred in a further oxidation step of one of the sulfinyl enantiomer to sulfone. ${ }^{13}$ The titanium-binaphthol complex catalyzes not only the asymmetric oxidation but also the kinetic resolution process.

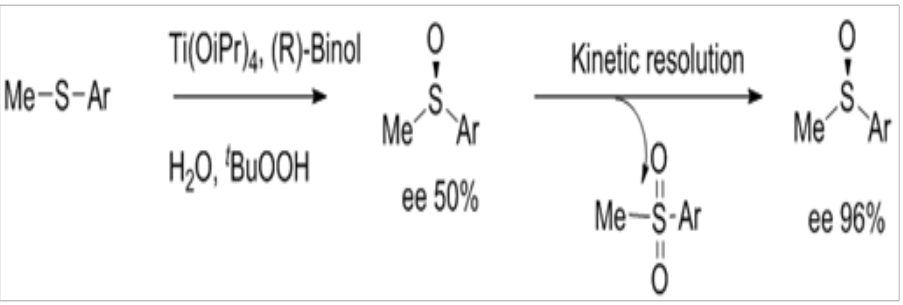

Figure I Asymmetric oxidation of the thioethers.
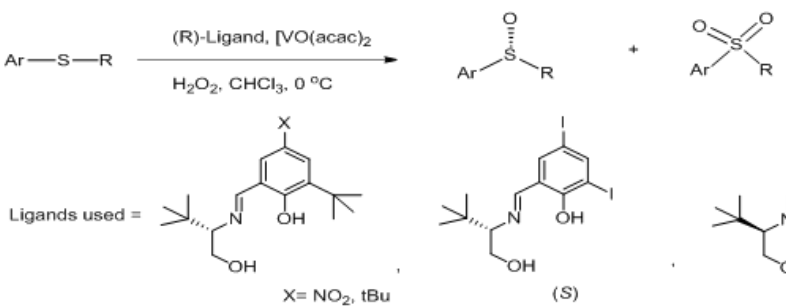

Figure 2 Catalytic approach to enantiomerically pure alkyl aryl sulfoxides using a combination of 3,5-diiodo Schiff.

More general are the application of the stoichiometric chiral oxidizing reagents described by Davis et al. ${ }^{14,15}$ The enantiopure (camphorsulfonyl)oxaziridines and their 8,8-dichloro derivatives available in both antipodal form, afforded sulfoxides with ee up to $96 \%$.

Dargo et al. ${ }^{16}$ came up with a catalytic approach to enantiomerically pure alkyl aryl sulfoxides using a combination of 3,5-diiodo Schiff base ligands and $\left[\mathrm{VO}(\mathrm{acac})_{2}\right]$ as the catalyst in the presence of $\mathrm{H}_{2} \mathrm{O}_{2}$ as oxidant (Figure 2). This combination proved to be effective affording sulfoxides possessing high ee and in high yields. Both enantiomers 
of the ligand can be easily prepared on multi gram scale and can be stored for prolonged periods without any loss of optical purity.

\section{Nucleophilic substitution on chiral sulfur derivatives}

The most widely used approach to enantiomerically pure sulfoxides is the Anderson synthesis ${ }^{17,18}$ based on the nucleophilic substitution on diastereomerically pure $(S S)$-menthyl $p$-toluenesulfinate ${ }^{19}$ with Grignard reagents which occurs with full inversion of configuration at sulfur (Figure 3). The classical method has been extensively used to prepare $p$-tolyl alkyl or aryl sulfoxides. The usefulness of this method is mainly due to the accessibility of the sulfinylating agent, obtained as mixture of sulfur epimers through esterification of $p$-toluenesulfinyl chloride. This originally described procedure to obtain enantiomerically pure sulfoxide was further improved and scaled up by equilibrating the epimeric sufinate in the presence of $\mathrm{HCl}$ to displace the equilibrium by precipitation of the $(S, S)$ diastereomer in acetone being thus isolating it in $80 \%$ yield.

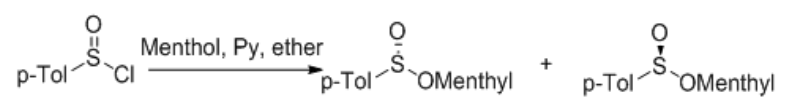

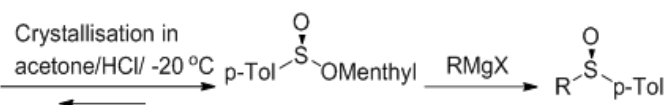

$[R=$ alkyl, aryl $]$

Figure 3 Nucleophilic substitution on chiral sulfur derivatives.

\section{Reactions exploiting sulfur chirality}

\section{As a chiral auxiliary in the diastereoselective reduction of $\beta$-ketosulfoxides}

Acyclic $\beta$-keto sulfoxides are readily available by the reaction of $\alpha$-sulfinyl anion with an ester. ${ }^{20,21}$ The main contribution to the synthesis of optically active secondary carbinols from $\beta$-keto sulfoxide is due to Carreno et al. ${ }^{22}$ The Stereochemical outcome in the reduction of the $\beta$-keto sulfoxide can be controlled by the configuration of the sulfoxide, the reducing agent and the absence or presence of a Lewis acid. The reduction of $\beta$-keto sulfoxide $\left(\mathrm{R}_{\mathrm{S}}\right) 1$ with DIBAL gave $\left(R_{S} S\right)$-carbinol 2 via intramolecular hydride transfer through a six-membered cyclic transition state (I), while the reduction with $\mathrm{LiAlH}_{4}$ or DIBAL in the presence of $\mathrm{ZnCl}_{2}$ as a Lewis acid give the epimer at the hydroxy center $\left(R_{S}, R\right)$-carbinol 3 which is rationalized by a conformationally rigid six-membered cyclic transition state (II) involving chelation of the Lewis acid to the sulfinyl and carbonyl oxygen (Figure 4). This methodology has been employed in enantioselective synthesis of methyl carbinol, 1,2-diols and epoxides which are used as the key intermediates in the total synthesis of natural products.

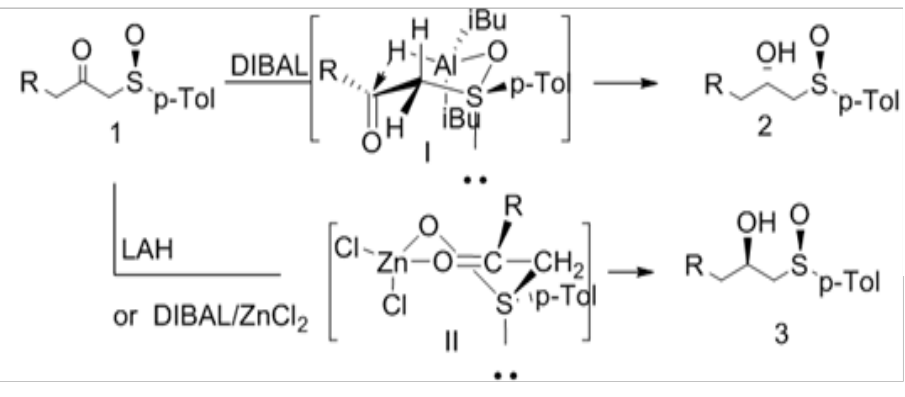

Figure 4 As a chiral auxiliary in the diastereoselective reduction of $\beta$-ketosulfoxides.
Methyl carbinol: One of the great advantages of sulfoxides is allowing the asymmetric induction step to be carried out at the very last part of the synthesis via this stereoselective $\beta$-keto sulfoxide reduction. A convergent synthesis of dimethyl ether (S)-6, ${ }^{23}$ a precursor of zearalenone, a naturally occurring macrolide with anabolic and uterotropic properties has been described (Figure 5). The enantiomerically pure hydroxy sulfoxide 4 derived from glutaric anhydride was transformed to the silyl ether of methyl carbinol 5 via silyl ether protection and subsequent desulfurization, which was further elaborated to the natural product.

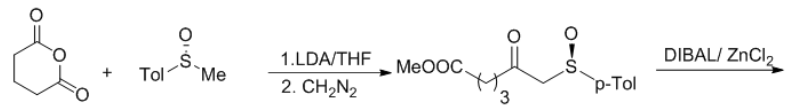

$$
\begin{aligned}
& \text { MeOOC }
\end{aligned}
$$

Figure 5 Methyl carbinol.

The anomeric effect was used to advantage in the highly stereoselective cyclization of dihydroxy disulfoxide 8 , accessible with both the $S$ and $R$ configuration at both the hydroxylic centers depending on the reduction method utilized in its obtention from the diketone 7. Upon treatment in acidic medium, compound 8 evolved into a spiroketal derivative whose desulfurization afforded compound 9, present in the rectal glandular secretion of certain species of fruit flies (Figure 6). ${ }^{24}$ The enantiomer could be synthesized from the dihydroxy disulfoxide resulting from reduction with DIBAL $/ \mathrm{ZnCl}_{2}$.

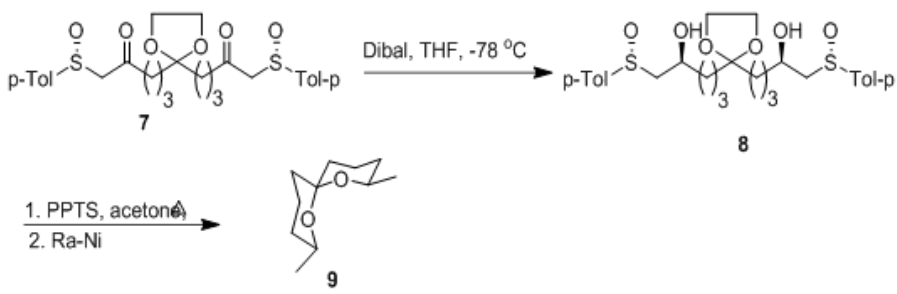

Figure 6 Enantiomer could be synthesized from the dihydroxy disulfoxide resulting from reduction with DIBAL/ZnCl .

1,2-Diols: The protocol transforming $\beta$-hydroxy sulfoxides into terminal 1,2-diols via the Pummerer rearrangement followed by desulfurization or LAH reduction of the resulting hemi-thioketal, has been utilized in the synthesis of several polyhydroxylated natural products. As a showcase, enantiomerically pure $\beta$-hydroxy unsaturated sulfoxide 10 was transformed to the triol derivative 11 by dihydroxylation. Compound 11 was further converted to L-penta$O$-acetylarabinitol 12 by applying the above strategy (Figure 7). ${ }^{25,26}$

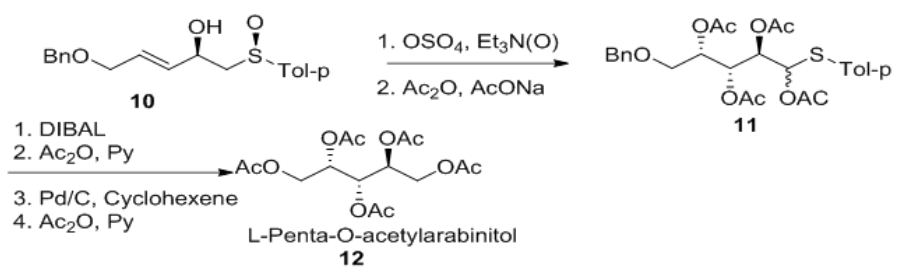

Figure 7 I,2-Diols.

Epoxides: $\beta$-Hydroxy sulfoxides can also be easily transformed into chiral epoxides giving access to a variety of natural products. The asymmetric synthesis of juvenile hormone II 13 was based on 
the diastereoselective alkylation and carbonyl reduction of $\beta$-keto sulfoxide $14 .{ }^{27}$ Alkylation of 14 produced a $(9: 1)$ mixture of $\left(\mathrm{R}, \mathrm{R}_{\mathrm{s}}\right)$ and $(\mathrm{S}, \mathrm{R})$ whose diastereoselective reduction afforded $\beta$ - hydroxy sulfoxide 15 which can be transformed to the target by routine synthetic procedures, (Figure 8).
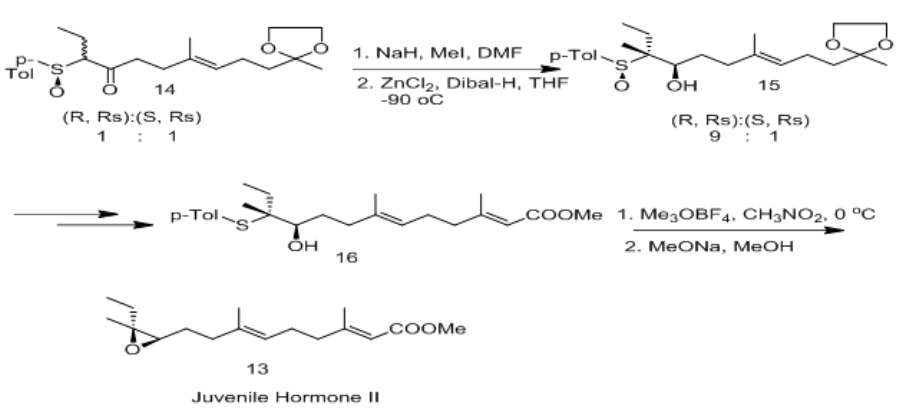

Figure 8 C. Epoxides.

\section{Conjugate additions to $\alpha, \beta$-unsaturated sulfoxides}

C-C bond formation: Sulfinyl butenolide (S)-17 was used as a Michael acceptor in the synthesis of optically pure 3-substituted butyrolactones. Zinc-bromide promoted benzylic Grignard reagent addition to (S)-17 led, after desulfurization and enolate acylation, to the anticancer agent (-)-podorhizon (18) (Figure 9). ${ }^{28}$

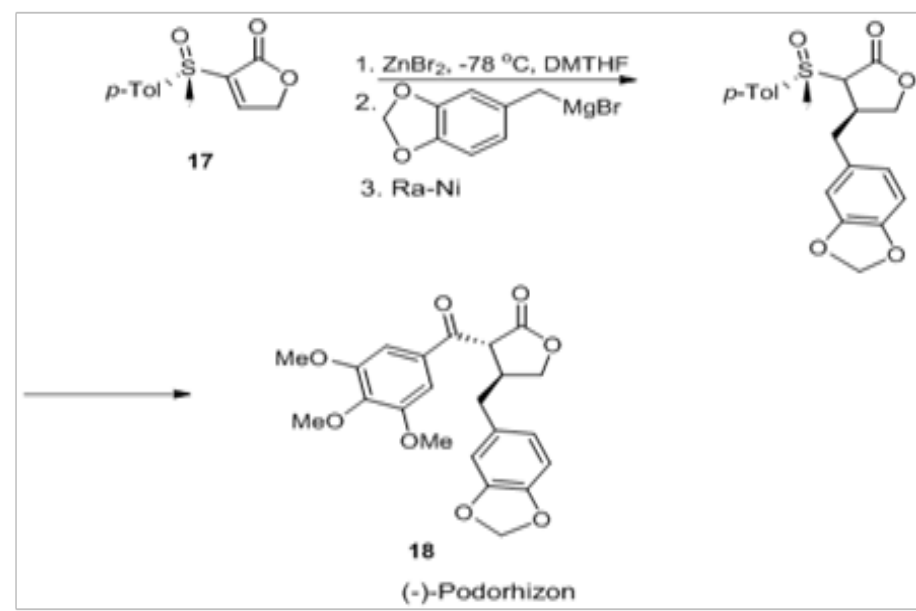

Figure $9 \mathrm{C}-\mathrm{C}$ bond Formation.

C-N bond formation: The total synthesis of (R)-carnegine 21 reported by Pyne and coworkers ${ }^{29}$ was based on the formation of the tetrahydroisoquinoline ring 20 upon cyclisation of (Z)-vinyl sulfoxide 19 under basic conditions. Desulfurization of 20 gave the alkaloid 21 (Figure 10).<smiles></smiles>

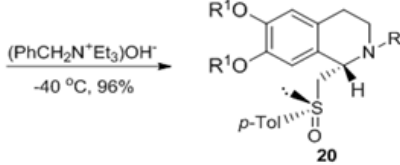

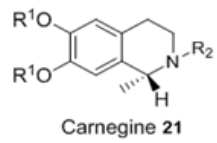

Figure 10 C-N Bond Formation.

\section{Reactions of sulfinyl carbanions}

1,2-Addition to carbonyl: Although the addition of simple $\alpha$-sulfinyl carbanions to carbonyl compounds proceeds with poor diastereoselectivity, the reaction was used as the key step in the synthesis of $(+)$-disparlure ${ }^{30} 25$ the pheromone produced by female gypsy moth (Porthetria dispor).

After chromatographic separation of the mixture of hydroxyl sulfoxides resulting from the reaction of lithium anion derived from optically pure sulfoxide 22 and undecanal, disparlure 25 , was obtained from the major diastereomer through a sequence involving sulfoxide reduction and base promoted cyclization of the sulfonium salt derived from the thioether (Figure 11).

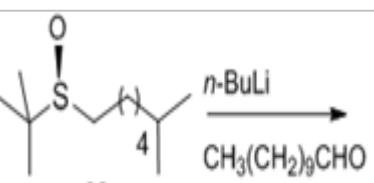

22<smiles>CC(C)N[C@@H](C(C)C)S(=O)O</smiles><smiles>C=CC(C)C(C)C1OC1C(C)=O</smiles>

25

Figure I I I,2-Addition to carbonyl.

Additions to $\boldsymbol{\alpha}, \boldsymbol{\beta}$-unsaturated compounds: The diastereoselective addition of simple $\alpha$-sulfinyl carbanions to $\alpha, \beta$-unsaturated compounds was reported by Scolastico who described the obtention of prostaglandin intermediate. The conjugate addition of the anion derived from (S)-dithioketal S-oxide 26 to 2-substituted cyclopentenone 27 in the presence of HMPT gave a 52:48 mixture of $\mathrm{C}-\alpha$ epimers that were converted into the enantiomerically pure protected aldehyde 28 by desulfurization. The free aldehyde has been used as starting material in prostanoids synthesis (Figure 12). ${ }^{3}$
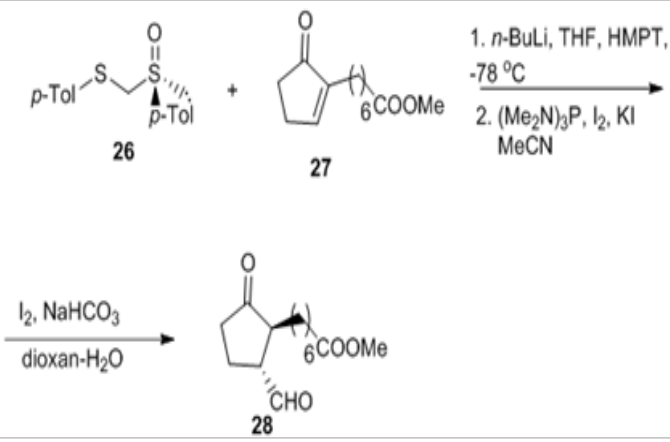

Figure 12 Additions to $\alpha, \beta$-unsaturated compounds.

The regio- and Stereochemical course of the reaction between ambident sulfinyl allyl anions and cyclic enones has been deeply studied by Binns et al..$^{32-34}$ while its application to asymmetric synthesis of natural products was mainly due to Hua et al. ${ }^{35,36}$ Total synthesis of (+)-hirsutene 32 employed two crucial reactions i.e.

i. An addition of ambident sulfinyl allyl anion of 29 to 2-methylcyclopentenone 30. The reaction occurred with high diastereocontrol. The step was used as the key to fix C-8 stereogenic center and 
ii. An efficient heterolytic ring closure involving vinyl thioether moiety to afford the $\mathrm{B}$ and $\mathrm{C}$ rings in 31 which was further elaborated to the target 32 (Figure 13). ${ }^{37}$

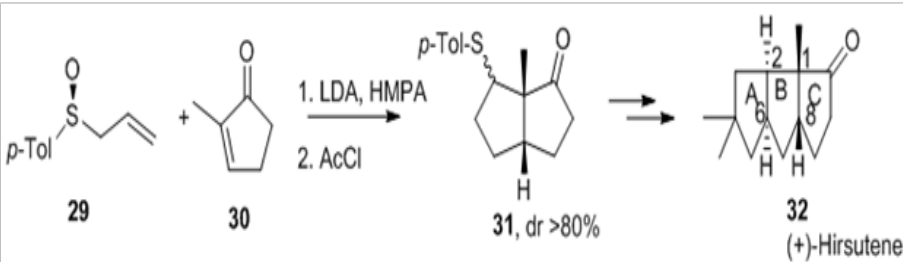

Figure I3 Regio- and stereochemical course of the reaction between ambident sulfinyl allyl anions and cyclic enones

Addition to $\mathbf{C}=\mathbf{N}$ of imines and sulfinyl imines: The nucleophilic addition of enantiomerically pure $\alpha$-sulfinyl carbanions to the diasterotopic $\mathrm{C}=\mathrm{N}$ double bond of imines allows the asymmetric synthesis of $\beta$-amino-sulfoxides. Bravo et al. was the first to establish that the addition of chiral sulfoxide-stabilized carbanion to $\mathrm{N}$-( $p$ methoxyphenyl)aldimines 34 bearing trifluromethyl, pentafluoroethyl and $\omega$-hydrotetrafluoroethyl groups afforded the corresponding enantiomerically enriched amine ${ }^{38} 35$ with excellent diastereocontrol (Figure 14).

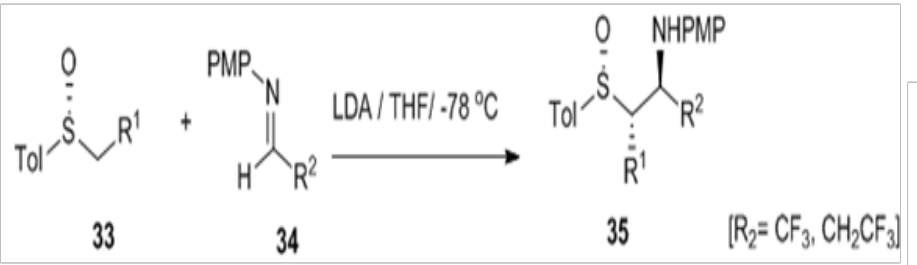

Figure 14 Addition to $\mathrm{C}=\mathrm{N}$ of imines and sulfinyl imines.

The Stereochemical outcomes of these reactions were rationalized assuming a kinetic control and an enantiodirecting effect of the fluoroalkyl group. The reaction has been extended to N-(PMP)arylimines, a practical way to prepare enantiopure $\alpha$-arylglycinols. ${ }^{39}$

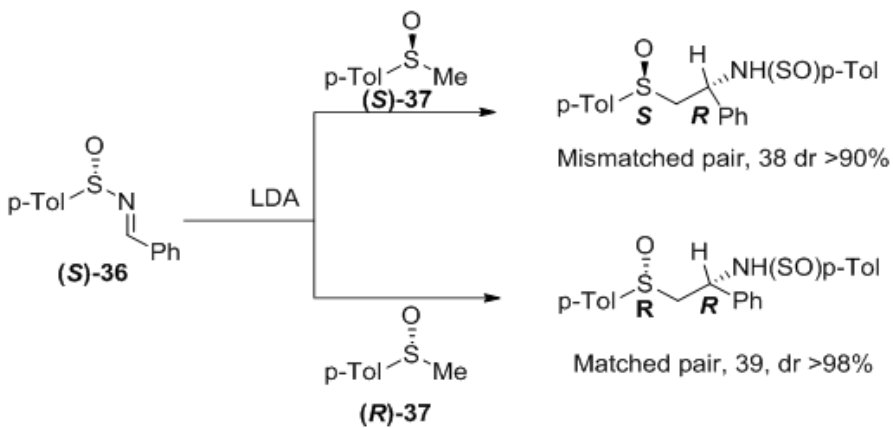

Figure I 5 The Stereoselectivity of the reaction is primarily controlled by the sulfur configuration of the starting electrophilic sulfinamide 36 .

The behavior of enantiomerically pure sulfinylimines with chiral $\alpha$-sulfinyl carbanions has been reported by Garcia Ruano et al. ${ }^{40}$ The highest Stereoselectivity is achieved when the configuration at the sulfur atoms of the two reagents are opposite (matched pair), affording only one diastereoisomer (Figure 15). For each pair, the major diastereoisomer has identical configuration at the new stereogenic carbon $(R$ in both case), despite the opposite configuration of the sulfoxide used as nucleophile in each case. This suggests that the stereoselectivity of the reaction is primarily controlled by the sulfur configuration of the starting electrophilic sulfinamide 36 .

\section{Pummerer rearrangement}

The reaction discovered by Pummerer, ${ }^{41}$ which brings about the transformation of sulfoxides bearing $\alpha$-hydrogen into $\alpha$-acyloxy sulfides upon treatment with acid anhydrides, has been widely used in synthesis (Figure 16). From the stereochemical point of view, this is a self-immolative asymmetric process where the chirality at sulfur is transferred to the $\alpha$-carbon. Unfortunately, the extent of asymmetric induction reported for the classical Pummerer rearrangement by different authors never exceeded $30 \%$ ee.

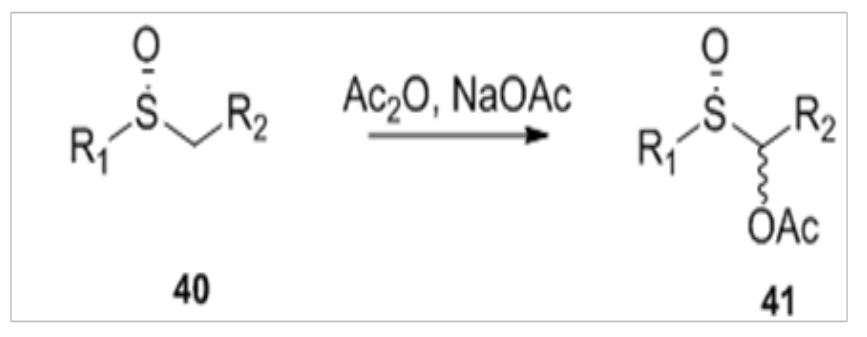

Figure- 16 Pummerer rearrangement.

Pummerer-type conditions have been used successfully for the stereoselective synthesis of $\beta$-lactam 43 , a precursor of some penicillin antibiotics. Trimethylsilyl triflate promoted Pumerer reaction of sulfoxide 42 afforded the lactam 43 (Figure 17). ${ }^{42}$

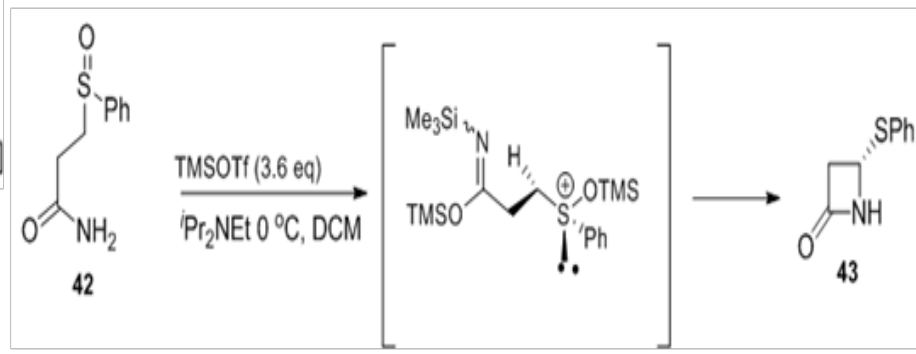

Figure 17 Trimethylsilyl triflate promoted Pumerer reaction of sulfoxide 42 afforded the lactam 43.

Pummerer-type conditions have been used successfully for the stereoselective synthesis of cyclic compounds by an intramolecular attack of a nucleophile on the sulfenium intermediate formed under the reaction conditions. The use of additive Pummerer-type rearrangement on cyclopentenone 44 allowed a short synthesis of methyl jasmonate $46,{ }^{43}$ a perfume essence (Figure 18). The dichlorolactone 45 was transformed to the natural product but with moderate Stereoselectivity ( $20 \%$ ee). A formal synthesis of (-)-serriconine 51 the sex pheromone of the cigarette beetle was recently reported from butyrolactone $48 .{ }^{44}$

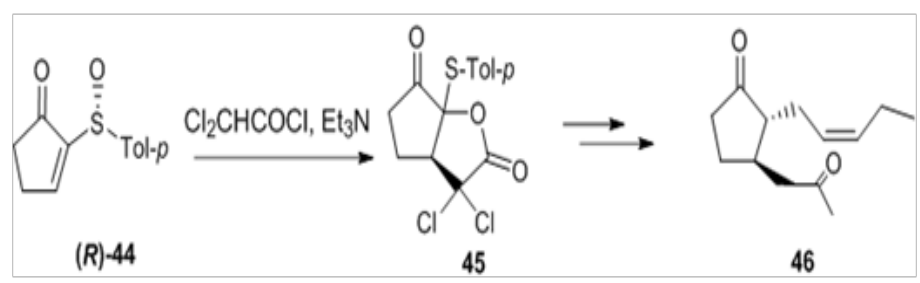

Figure 18 Dichlorolactone 45 was transformed to the natural product but with moderate Stereoselectivity.

The starting vinyl sulfoxide 47 reacted with dichloroketene and after aluminium amalgam and raney nickel treatment, 48 was obtained as a single diastereomer and enantiomer. The intermediate $\delta$-lactone 
50 which had been already transformed into the natural product was synthesized after reduction of 48 and homologation of the diol followed by hydrolysis and lactonization (Figure 19).

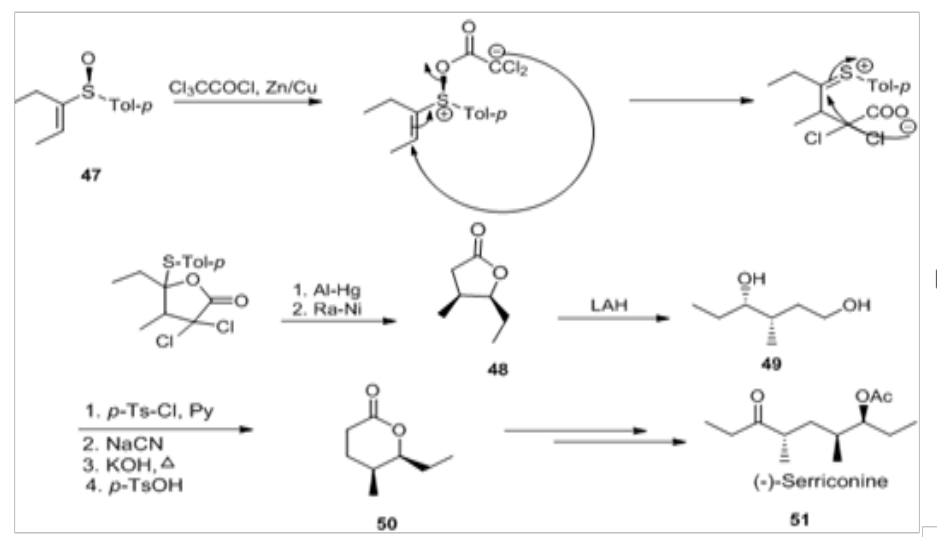

Figure 19 Intermediate $\delta$-lactone 50 which had been already transformed into the natural product was synthesized after reduction of 48 and homologation of the diol followed by hydrolysis and lactonizat $\mathrm{I}$.

Bravo et al. reported an unusual Pummerer rearrangement of $\gamma$-trifluoro- $\beta$-aminosulfoxides. ${ }^{45}$ surprisingly; treatment of a $\beta$-aminosulfoxide 52 with TFAA and sym-collidine produced a sulfenamide 57 in good yield as the only product (Figure 20). This "abnormal" rearrangement product was successfully converted to the corresponding $\beta$-fluoro- $\alpha$-aminoalcohol.

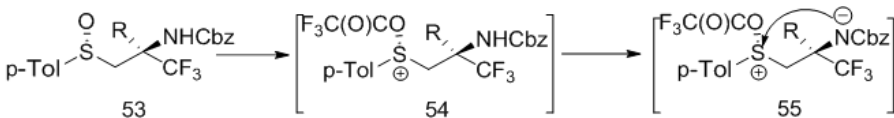

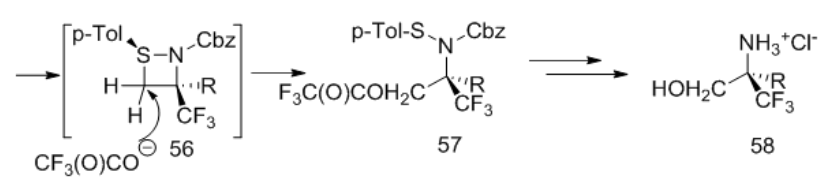

Figure 20 "Abnormal" rearrangement product was successfully converted to the corresponding $\alpha$-fluoro- $\beta$-aminoalcohol.

\section{Reaction involving vinyl sulfoxides}

Cycloaddition reactions: The combination of a Diels-Alder represents a very powerful method for $\mathrm{C}-\mathrm{C}$ bond formation in a stereocontrolled manner. The sulfinyl group has equally become one of the most interesting chiral inductors in asymmetric Diels-Alder reactions, due to

a. Its ability to differentiate between diastereotopic faces of neighboring double bonds

b. The ease of chemical transformations into different functional groups including its clean removal under mild conditions and

c. The existence of well behaved methods for preparing enantiomerically pure sulfoxides. ${ }^{46}$

A. Sulfinyl dienophiles: Application of optically active vinyl sulfoxides as dienophiles is a fascinating strategy, since the chiral sulfinyl auxillary is known to exert a high asymmetric induction in the carbon-carbon bond formation. ${ }^{47}$ An enantioselective synthesis of (+)-royleanone 62, an insecticide and disinfectant agent, could be developed using the sulfinylquinone methodology. The key step was a tandem asymmetric Diels-Alder reaction/pyrolytic sulfoxide elimination process involving (S)-3-hydroxy-2-isopropyl-5tertbutylsulfinyl-p-benzoquinone 59 as chiral auxillary (Figure 21). ${ }^{48}$ The asymmetric Diels-Alder reaction of an enantiomerically pure sulfinyl naphthoquinone 63 and a vinyl cyclohexenol 64 was used as the key step to establish the tetracyclic angucyclinone skeleton 65. The sulfinyl group proved to achieve a double induction in the Diels-Alder reaction which led to an efficient kinetic resolution of the racemic diene (Figure 22). ${ }^{49}$

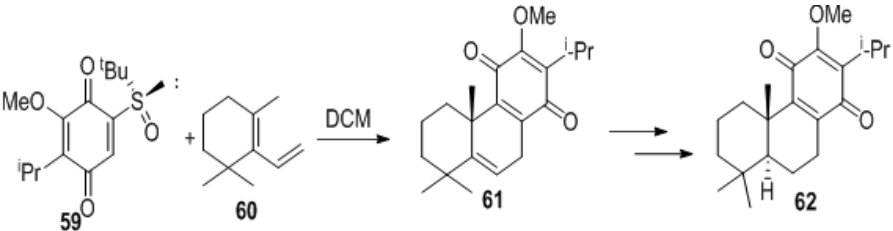

(+)-Royleanone

Figure 2 I Sulfinyl dienophiles.<smiles></smiles>

63

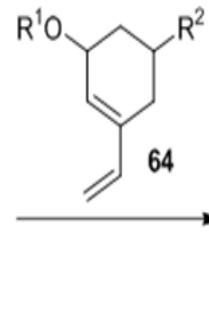

64

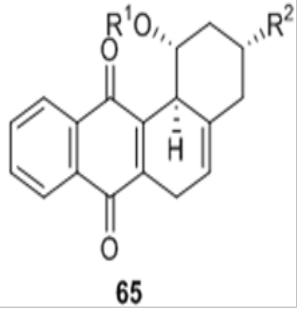

Figure 22 The sulfinyl group proved to achieve a double induction in the Diels-Alder reaction which led to an efficient kinetic resolution of the racemic diene.

B. Sulfinyl dienes: Diels-Alder reactions of enantiomerically enriched sulfinyl dienes are less well documented in the literature, presumably due to the synthetic difficulties in preparing such molecules. Nevertheless, enantiomerically enriched sulfinyl 1,3-butadienes are frequently used to react with a variety of dienophiles that undergo Diels-alder cycloaddition. The use of a Lewis acid to restrict the rotation around the C-S bond has been used to improve the Stereoselectivity. Enantiomerically pure hydroxyl sulfinyl dienes 66 were prepared from base- induced rearrangement of epoxy vinyl sulfoxides, the E-Z- stereoselectivity being controlled by the sulfur auxillary.

These compounds were used in a Diels-Alder reaction with N-phenylmaleimide 67 and phenyltriazolinedione 68 and were found to react with high facial selectivity. Interestingly, the sulfinyl group managed to override the intrinsic allylic stereocontrol of the substrates (Figure 23). ${ }^{50}$

Complete regioselectivity and Stereoselectivity were observed for the first asymmetric hetero Deils-Alder reaction of 1-sulfinyl dienes with acylnitroso derivatives such as benzyl nitrosoformate. The stereochemical course of the reaction could be explained by considering that the heterodienophile approached the less hindered face of diene that which supported the lone electron pair at sulfur, with the sulfinyl group in an s-trans arrangement with respect to $\mathrm{C} 1=\mathrm{C} 2$ (Figure 24$)$.

The asymmetric Diels-Alder reaction of 2-sulfinyl dienes has been used in the total synthesis of (-)-(1S, 5R)-Karahana ether $74^{51}$ (Figure 20). The key step involves the cycloaddition of maleic anhydride 76 with 2-sulfinylpentadiene 75 which proceeds in good yield to afford a 
4:1 mixture of diastereomeric cycloadducts, which was elaborated to the monoterpenoid (Figure 25).

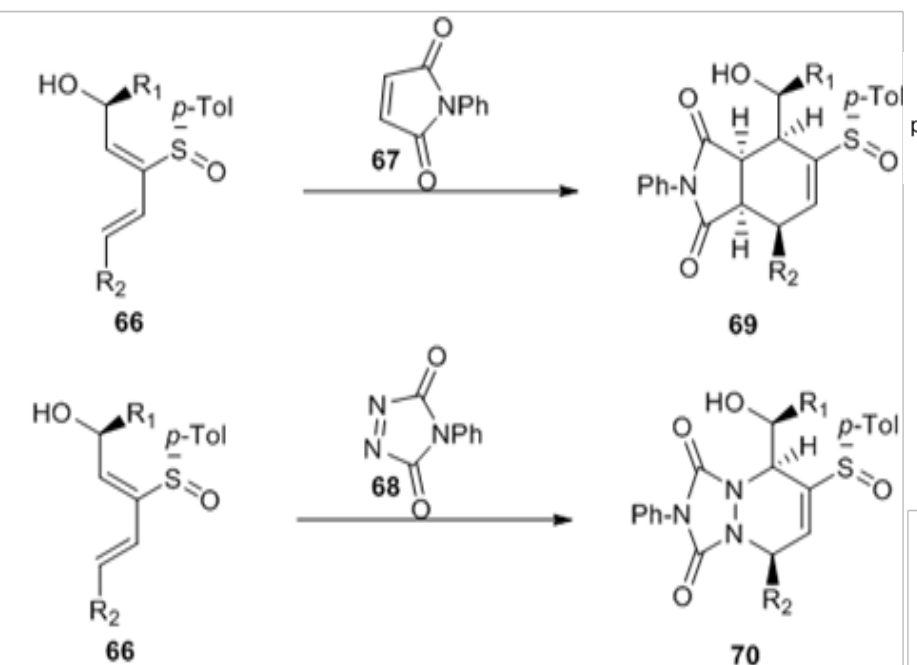

Figure23 Sulfinyl dienes.

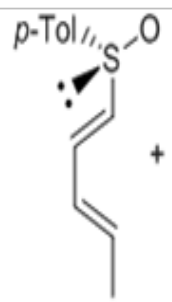

71

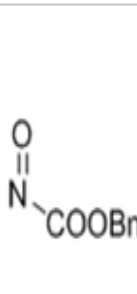

72

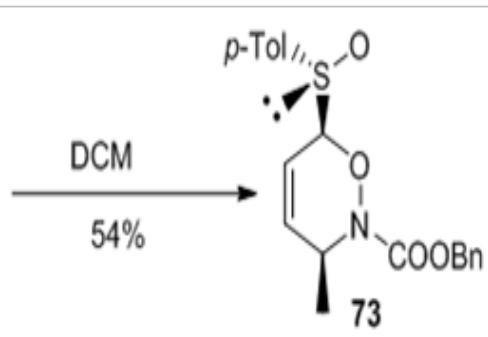

de $=100 \%$

Figure 24 The stereochemical course of the reaction could be explained by considering that the heterodienophile approached the less hindered face of diene that which supported the lone electron pair at sulfur, with the sulfinyl group in an s-trans arrangement with respect to $\mathrm{Cl}=\mathrm{C} 2$.

$\overbrace{75}^{\text {P-Tol }}$

75

Figure 25 The key step involves the cycloaddition of maleic anhydride 76 with 2-sulfinylpentadiene 75 which proceeds in good yield to afford a 4:I mixture of diastereomeric cycloadducts, which was elaborated to the monoterpenoid.

Similarly, hetero-Diels-Alder reaction of the diene 79 with benzyl nitrosoformate $72^{52}$ takes place at low temperature to afford the cycloaddition product in 54\% yield as a single diastereoisomer. This adduct 80 was transformed into enantiomerically pure 1,4,5-trideoxy-1,4-imino-L-ribitol 81 (Figure 26).<smiles>CC(C)(C)OC(=O)N=O</smiles>

Figure 26 Adduct 80 was transformed into enantiomerically pure I,4,5-trideoxy-I,4-imino-L-ribitol 81.

Addition reactions: Hydride reduction of the enamine with L- and K-Selectride displayed high diastereoselectivity (dr 95\%) (Figure
27). The resulting compounds can be desulfinylated under reducing or Pummerer conditions to afford enantiomerically pure amines, aminoalcohols or aminoacids.

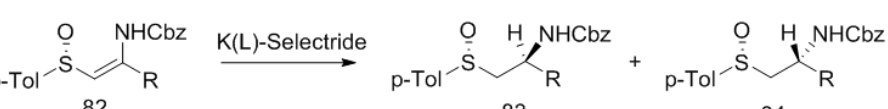
82

83 84

Figure 27 Addition reactions.

Takeda et al. have described a reductive cyclization to form functionalized cyclohexane derivatives that involves an intramolecular Michael addition. ${ }^{53}$ Hydride reduction of an enolate 85 afforded an ester enolate, which underwent intramolecular conjugate addition with vinylsulfoxide moiety to yield trans cyclization product 86 (Figure 28).

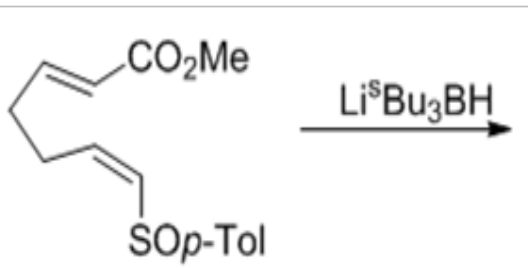

85

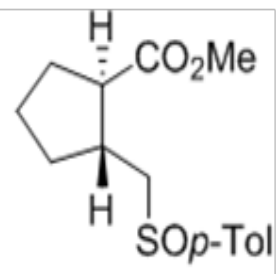

86
Figure 28 Hydride reduction of an enolate 85 afforded an ester enolate, which underwent intramolecular conjugate addition with vinylsulfoxide moiety to yield trans cyclization product 86.

Enantiomerically enriched sulfinyl auxiliaries are able to induce asymmetry in nucleophilic substitution reactions. Marino et al. ${ }^{54}$ demonstrated highly regio- and stereoselective $\mathrm{S}_{\mathrm{N}}{ }^{2}$ reactions of enantiomerically enriched epoxy vinyl sulfoxide 87 with alkyl cyanocuprates to afford the hydroxy sulfoxides 88 and 89 (Figure 29). The displacements can either take place in syn or anti fashion, in which the sulfoxide is the predominant element of stereocontrol.

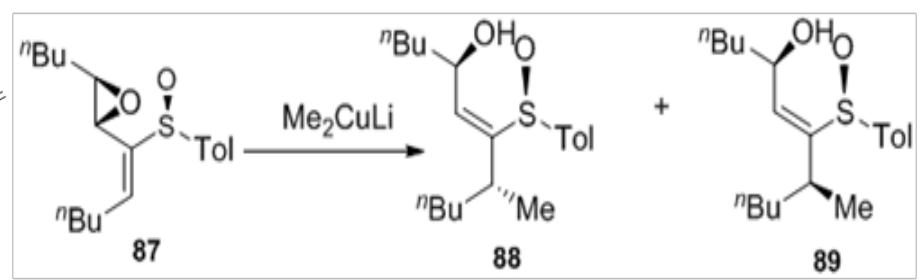

Figure 29 The displacements can either take place in syn or anti fashion, in which the sulfoxide is the predominant element of stereocontrol.

Steroid 11-oxoequilenin methyl ether $90^{55}$ was synthesized by stereospecific addition of the large (6-methoxy-2-naphthy1) magnesium bromide 91 to (S)-92 followed by trapping of the enolate intermediate with methyl iodide to yield 93 (Figure 30). Further elaboration gave optically pure $(S, S)-90$.

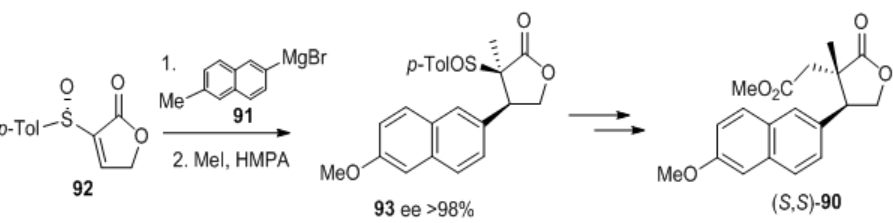

Figure 30 Steroid II-oxoequilenin methyl ether $90^{42}$ was synthesized by stereospecific addition of the large (6-methoxy-2-naphthyl) magnesium bromide 91 to (S)-92 followed by trapping of the enolate intermediate with methyl iodide to yield 93. 
While the intermolecular conjugate addition of nitrogen nucleophiles to $\alpha, \beta$-unsaturated sulfoxides ${ }^{56}$ proceeds very slowly, the intramolecular Michael addition took place at lower temperature with higher reaction rates ${ }^{57,58}$ and modest asymmetric induction.

Compound 95, a key intermediate in the synthesis of $\alpha$-tocoferol $91^{59}$ (vitamin E), was obtained by the addition of lithioalkenyl sulfoxide 92 to aromatic aldehyde 93 followed by intramolecular ring closure (Figure 31). A sole diastereomer was detected in the first reaction as well as in the intramolecular cyclization which took place via $\mathrm{S}_{\mathrm{N}} 2$ mechanism in a syn stereo specific fashion to give (2S)-95.<smiles>[R20]Oc1c(C)cc([R20])c(C)c1C=O</smiles>

$\mathrm{R}^{1}=\left(\mathrm{CH}_{3} \mathrm{O}\right)_{2} \mathrm{CH}$ $R^{2}=T B S$

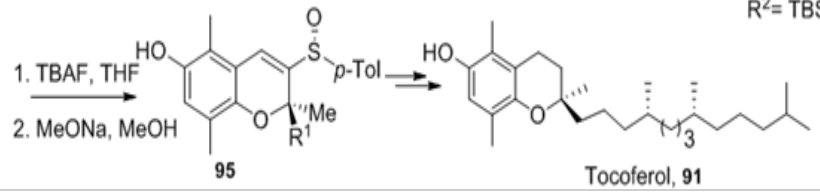

Figure 3I A sole diastereomer was detected in the first reaction as well as in the intramolecular cyclization which took place via $\mathrm{S}_{\mathrm{N}} 2$ mechanism in a syn stereospecific fashion to give (2S)-95.

A. Sigmatropic rearrangements: Until very recently, no accounts were known of sigmatropic rearrangements involving the double bond of vinylic sulfoxides. Metzner et al. pioneered in this area and published the first Claisen rearrangement that was stereocontrolled by the sulfinyl group. ${ }^{60,61}$ Ketene dithioacetal 96 derived from dithioester 97 underwent facile rearrengments at room temperature into $\gamma$-unsaturated $\alpha$-sulfinyl dithioesters 98 with excellent diastereoselectivity ( $\mathrm{dr}>99 \%$ ) (Figure 32 ).
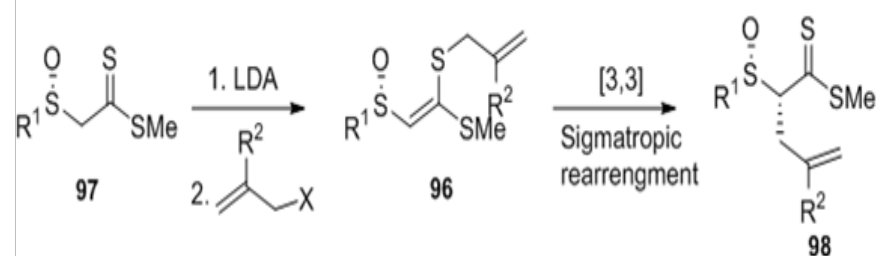

$\mathrm{R}^{1}=\mathrm{Me},{ }^{\prime} \mathrm{Bu},{ }^{\prime} \mathrm{Pr}, \mathrm{C}_{6} \mathrm{H}_{1}$ $R^{2}=H, M e$

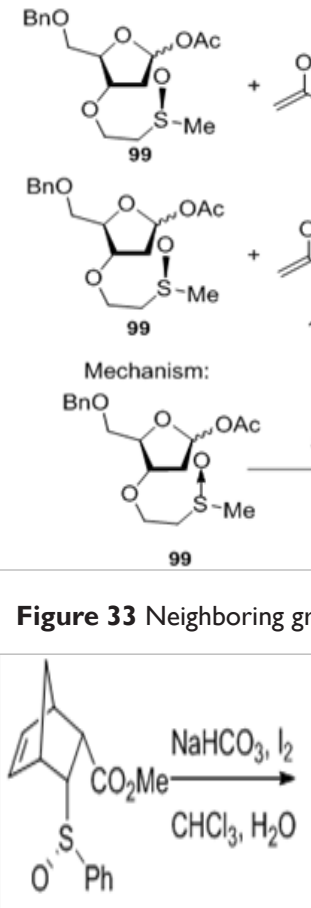

104

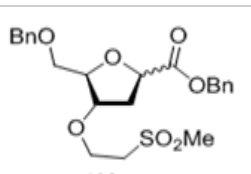

2. [O]<smiles>COCCOC[C@@H]1O[C@H](C(C)=O)C[C@@H]1OC</smiles>

1012103

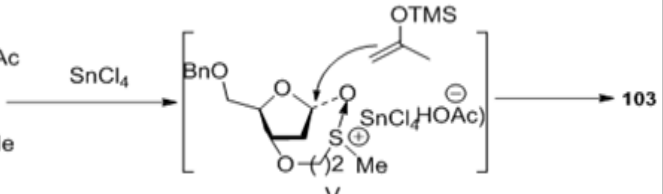
$\mathrm{V}$

Figure 33 Neighboring group participation of sulfoxides.

Figure 34 Sulfinyl oxygen as a nucleophile in cyclic systems.

Sulfinyl oxygen as a nucleophile in acyclic systems: Sulfoxides have been used as pendant nucleophiles in the hydrolysis of epoxy sulfoxide 106 to dihydroxy sulfoxide 107 . The sulfoxide delivered the oxygen intramolecularly (VII) to open the epoxide with high degree of regiocontrol. ${ }^{64}$ The reaction was postulated to proceed via the five membered cyclic sulfoxonium salts, which was hydrolysed to yield the dihydroxy sulfoxide (Figure 35).

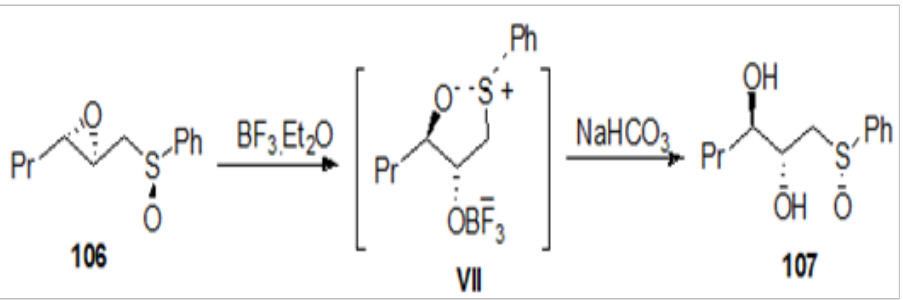

Figure-35 Sulfinyl oxygen as a nucleophile in acyclic systems.

Sulfinyl group participation was also demonstrated by Horner et al. ${ }^{65}$ in the stereoselective bromohydroxylation of 1,2-allenylsulfoxides with $\mathrm{Br}_{2}$ and $\mathrm{H}_{2} \mathrm{O}$ (Figure 36).

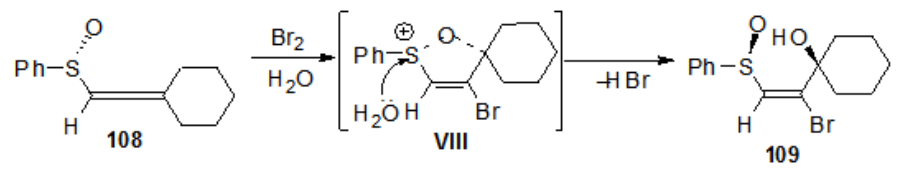

Figure 36 Sulfinyl group participation was also demonstrated by Binder et al. ${ }^{50}$ in the stereoselective bromohydroxylation of I,2-allenylsulfoxides with $\mathrm{Br}_{2}$ and $\mathrm{H}_{2} \mathrm{O}$.

The potential of the sulfoxide group to act as an intermolecular nucleophile was demonstrated by David et al. ${ }^{66} \mathrm{~A}$ number of olefins were converted to bromohydrins stereospecifically by the use of 
N-bromosuccinimide (NBS) and moist dimethylsulfoxide (DMSO) (Figure 37).

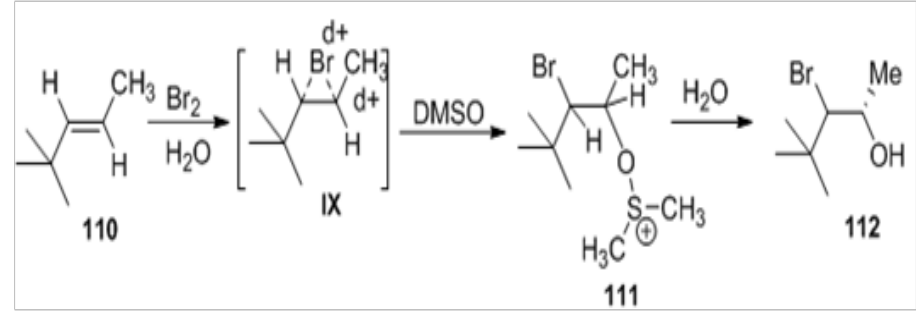

Figure 37 A number of olefins were converted to bromohydrins stereospecifically by the use of N-bromosuccinimide (NBS) and moist dimethylsulfoxide (DMSO).

\section{Conclusion}

With few exceptions, the sulfoxide group acts as an efficient chiral inducer in a number of organic transformations and has potential applications in natural product synthesis. ${ }^{67-69}$ The key to the success is related to the steric and electronic differences between the substituents at the sulfur, as well as the conformational behavior of the sulfinyl group, which is able to react through a rigid conformation. In many cases use of Lewis acid could dramatically alter the stereoselectivity of the reaction. As, the sulfinyl functionality can co-ordinate both with its oxygen or sulfur atom extending the scope of metal used as catalyst. The field will probably be of growing interest in the near future.

\section{Acknowledgements}

All authors contributed toward data analysis, drafting and revising the paper and agree to be accountable for all aspects of the work.

\section{Conflict of interest}

There is no conflict of interest.

\section{References}

1. Mikolajczyk M, Drabowicz J. Topics in Stereochem. 1982;13:435.

2. Davis A, Chen BC. Asymmetric synthesis of amino acids using sulfinimines (thiooxime S-oxides). Chem Soc Rev. 1998;27(1):13-18.

3. Davis FA, Portonovo PS, Reddy RE et al. Asymmetric Synthesis using Sulfinimines (Thiooxime S-Oxides). Phosph Sulf Silic and related comp. 1997;120(1):291-303.

4. Barbachyn JD, Johnson CR. In Asymmetric Synthesis. Morisson JD, Scott JW, editors. NY: Acad Press; 1983:4:227.

5. Solladié G. Comprehensive Organic Synthesis. In: Trost BM, Fleming I, editors. Oxford: Pergamon Press; 1991:6(3).

6. Mislow K, Siegel J. Stereoisomerism and local Chirality. J Am Chem Soc. 1984;106(11):3319-3328.

7. Solladie G. Asymmetric Synthesis Using Nucleophilic Reagents Containing a Chiral Sulfoxide Group. Synthesis. 1981;(3):85-196.

8. Pitchen P, Dunach E, Deshmukh NN, et al. An efficient asymmetric oxidation of sulfides to sulfoxides. J Am Chem Soc. 1984;06(26):8188-8193.

9. Di Furia F, Modena G, Seraglia R. Synthesis of Chiral Sulfoxides by Metal-Catalyzed Oxidation with t-Butyl Hydroperoxide. Synthesis. 1984;(4):325-326.

10. Zhao SH, Samuel 0, Kagan HB. Asymmetric oxidation of sulfides mediated by chiral titanium complexes: mechanistic and synthetic aspects. Tetrahedron. 1987;43(21):5135-5144.
11. Kagan HB, Rebiere F. Some Routes to Chiral Sulfoxides with Very High Enantiomeric Excesses. Synlett. 1990(11):643-650.

12. Komatsu N, Nishibayashi Y, Sugita T, et al. Catalytic asymmetric oxidation of sulfides to sulfoxides using R-(+)-binaphthol. Tetrahedron Lett. 1992;33(37):5391-5394.

13. Komatsu N, Hashizume M, Sugita T, et al. Catalytic asymmetric oxidation of sulfides to sulfoxides with tert-butyl hydroperoxide using binaphthol as a chiral auxiliary. J Org Chem. 1993;58(17):4529-4533.

14. Davis FA, Reddy RT, Weismiller MC. (-)-.alpha.alpha.-Dichlorocampho rsulfonyloxaziridine: a superior reagent for the asymmetric oxidation of sulfides to sulfoxides. J Am Chem Soc. 1989;111(15): 5964-5965.

15. Davis FA, Reddy RT, Han W, et al. Chemistry of oxaziridines. 17. $\mathrm{N}$-(Phenylsulfonyl)(3, 3-dichlorocamphoryl) oxaziridine: a highly efficient reagent for the asymmetric oxidation of sulfides to sulfoxides. J Am Chem Soc. $1992 ; 114(4): 1428-1437$.

16. Dargo C, Caggiano L, Jackson RF. Vanadium-catalyzed sulfur oxidation/ kinetic resolution in the synthesis of enantiomerically pure alkyl aryl sulfoxides. Angew Chem Int Ed Engl. 2005;44(44):7221-7223.

17. Andersen KK. Synthesis of (+)-ethyl-tolyl sulfoxide from (-)-menthyl (-)-p-toluenrsulfinate. Tetrahedron Lett. 1962;3(3):93-95.

18. Andersen KK, Gaffield W, Papanikolau NE, et al. Optically Active Sulfoxides. The Synthesis and Rotatory Dispersion of Some Diaryl Sulfoxides². J Am Chem Soc. 1964;86(24):5637-5646.

19. Solladie G, Hutt J, Girardin A. Improved Preparation of Optically Active Methyl p-Tolyl Sulfoxide. Synthesis. 1987;(2):173.

20. Corey EJ, Chaykowski M. The Pentalenyl Dianion. J Am Chem Soc. 1962;84(5):866-866.

21. Corey EJ, Chaykowski M. Methylsulfinyl Carbanion $\left(\mathrm{CH}_{3}-\mathrm{SO}-\mathrm{CH}_{2}-\right)$. Formation and Applications to Organic Synthesis. $\mathrm{J} \mathrm{Am} \mathrm{Chem} \mathrm{Soc.}$ 1965;87(6):1345-1353.

22. Carreno MC, Garcia Ruano JL, Martin AM, et al. Stereoselective reductions of 2-keto sulfoxides with hydrides. J Org Chem. 1990;55(7):2120-2128.

23. Solladie G, Maestro MC, Rubio A, et al. Asymmetric synthesis of (S)zearalenone dimethyl ether, an orsellinic acid type macrolides. J Org Chem. 1991;56(7):2317-2322.

24. Solladie, G, Huser N. Asymmetric synthesis of chiral spiroacetals from chiral diketodisulfoxides: 2,8-dimethyl-1,7-dioxaspiro [5,5] undecane. Tetrahedron: Asymmetry. 1994;5(2):255-260.

25. Solladie G, Hutt J, Frechou C. Asymmetric synthesis of polyhydroxylated natural products I. Efficient preparation of L-arabinitol. Tetrahedron Lett. 1987;28(1):61-64.

26. Solladie G, Frechou C, Hutt J, et al. BUZZ SOC Chim Fr. 1987;827.

27. Kosugi H, Kanno 0, Uda H. Tetrahedron: Asymmetry. 1994;5(7):1139-1142.

28. Posner GH, Kogan TP, Haines SR, et al. S)-(+)-2-(-tolylsulfinyl)-2buten-4-olide: an enantiomerically pure Michael acceptor for asymmetric synthesis of 3-substituted 4-butanolides. (-)-podorhizon. Tetrahedron Lett. 1984;25(25): 2627-2630.

29. Pyne SG, Bloem P, Chapman SL, et al. Chiral sulfur compounds. 9. Stereochemistry of the intermolecular and intramolecular conjugate additions of amines and anions to chiral (E)- and (Z)-vinyl sulfoxides. Total syntheses of (R)-(+)-carnegine and (+)- and (-)-sedamine. J Org Chem. 1990;55(3):1086-1093.

30. Farnum DG, Veysoglu T, Carde AM, et al. A stereospecific synthesis of (+)-disparlure, sex attractant of the gypsy moth. Tetrahedron Lett. 1977;18(46):4009-4012.

31. Colombo L, Gennari C, Resnati G, et al. Chiral acyl anion equivalents: asymmetric synthesis of 11-deoxy-ent-prostaglandin intermediates. $J$ Chem Soc Perkin Trans 1. 1981:1284-1286. 
32. Binns MR, Andrew A Katsifis, Haynes RK, et al. The diastereospecific aprotic conjugate addition reactions of carbanions derived from allylic sulfoxides and allylic phosphine oxides. Tetrahedron Lett. 1986;26(12):1565-1568.

33. Binns MR, Haynes RK, Katsifis AG, et al. J Am Chem Soc. 1988;110:5411.

34. Haynes RK, Katsifis AG, Vonwiller SC, et al. Aprotic conjugate addition of allyllithium reagents bearing polar groups to cyclic enones. 2. 2-Alkyl-, 2,3-dialkyl- and 1,3-dialkylallyl systems. J Am Chem Soc. $1988 ; 110(16): 5423-5433$.

35. Hua DH, Sinai-Zingde G, Venkataraman S. Asymmetric synthesis via chiral sulfinylallyl anion. Total synthesis of $(+)$-hirsutene: facile ring closure involving enol thioether and enol acetate moieties. $\mathrm{J} \mathrm{Am} \mathrm{Chem}$ SOC. 1985;107(13):4088-4090.

36. Hua DH, Venkataraman S, Ostrander RA, et al. Asymmetric synthesis of (+)-hirsutene. J Org Chem. 1988;53:507-515.

37. Hua DH, Venkataraman S, Coulter MJ, et al. Asymmetric induction in the addition reactions of chiral sulfinylallyl anions (ambident nucleophiles) with enones (ambident electrophiles). Ring closure of enol thioether ketones. J Org Chem. 1987;52(5):719-728.

38. Pyne SG, Dikic B. Diastereoselective kinetically and thermodynamically controlled additions of $(\mathrm{R})-(+)$-methyl p-tolyl sulphoxide anion to imines (tolyl $=\mathrm{C}_{6} \mathrm{H}_{4} \mathrm{Me}$ ). J Chem Soc Chem Commun. 1989;(13):826-827.

39. Bravo P, Capelli S, Crucianelli M, et al. Asymmetric synthesis of $\alpha$-arylglycinols via additions of lithium methyl p-tolyl sulfoxide to N-(PMP)arylaldimines followed by "non oxidative" Pummerer reaction. Tetrahedron. 1999;55(10):3025-3040.

40. Garcia Ruano JL, Alcudia A, del Prado M, et al. Additions of Enantiopure $\alpha$-Sulfinyl Carbanions to (S)-N-Sulfinimines: Asymmetric Synthesis of $\beta$-Amino Sulfoxides and $\beta$-Amino Alcohols. J Org Chem 2000;65(10):2856-2862.

41. Pummerer R. Über Phenylsulfoxy-essigsäure. (II.). Ber Dtsch Chem Ges. 1910;43(2):1401-1412.

42. Kaneko T, Okamoto Y, Hatada K. Asymmetric synthesis of $\beta$-lactams using a Pummerer reaction. J Chem Soc Chem Commun. 1987;(19):1511-1512.

43. Kita Y, Shibata N. Asymmetric Pummerer-Type Reactions Induced by O-Silylated Ketene Acetals. Synlett. 1996;(4):289-296.

44. Ferreira JTB, Marques JA, Marino JP. A formal and enantioselective synthesis of (-)-serricornin, the sex pheromone of the cigarette beetle (lasioderma serricorne F). Tetrahedron: Asymmetry. 1994;5(4):641-648.

45. Arnone A, Bravo P, Bruché, L, et al. Unusual non-oxidative pummere rearrangement of $\gamma$-trifluoro- $\beta$-aminosulfoxides. Tetrahedron Lett. 1995;36(40):7301-7304

46. Carretero JC, Garcia Ruano JL, Martin Cabrejas LM. Asymmetric DielsAlder reaction of $(\mathrm{S})$ - $\alpha$-p-tolylsulfinyl $\alpha, \beta$-unsaturated esters: the role of the sulfinyl group in asymmetric Diels-Alder reactions of vinylsulfoxides. Tetrahedron: Asymmetry. 1997;8(13):2215-2225.

47. Yuste F, Ortiz B, Perez JI, et al. ( \pm )-3,3-Diethoxycarbonyl-2-ptolylsulfinylacrylonitrile as dienophile: relationship between the barrier for pyramidal inversion of vinyl sulfoxides and the electronic effects of the substituents at the double bond. Tetrahedron. 2002;58(13):2613-2620.

48. Carrefio MC, Garcia Ruano JL, Urbano A. Asymmetric Diels-Alder reactions of (S)-2-(p-tolylsulfinyl)-1,4-naphthoquinones. J Org Chem. 1992;57(25):6870-6876.

49. Carreño MC, Urbano A, Fischer J. Enantioselective Diels-Alder Approach to Angucyclinones from (S)-2-(p-Tolylsulfinyl)-1,4-naphthoquinone and Substituted Racemic Vinylcyclohexenes*. Angew Chem Int Ed Engl. 1997;36(15):1621-1623.

50. Fernandez de la Pradilla R, Montero C, Roberto Fernández de la P, et al. Sulfinyl versus allylic stereocontrol in Diels-Alder cycloadditions of hydroxy 2-sulfinyl butadienes. Chem Commun. 1998;(3):409-410.

51. Gosselin P, Bonfand E, Maignan C. Application of the Stereoselective Diels-Alder Reaction of Enantiopure 2-Sulfinyl Dienes: Synthesis of (-)-(1S,5R)-Karahana Ether†. J Org Chem. 1996;61(25): 9049-9052.

52. Arribas C, Carreño MC, Garcia Ruano JL, et al. First Asymmetric Hetero Diels-Alder Reaction of 1-Sulfinyl Dienes with Nitroso Derivatives. ANew Entry to the Synthesis of Optically Pure 1,4-Imino-1-ribitol Derivatives. Org Lett. 2000;2(20):3165-3168.

53. Yoshizaki H, Tanaka T, Yoshii E, et al. Asymmetric Reductive Cyclization Using the Intramolecular Conjugate Addition of Enolates onto $\alpha, \beta$ Unsaturated Sulfoxides. Tetrahedron Lett. 1998;39(1-2):47-50.

54. Marrino JP, Anna LJ, Fernandez MV, et al. Sulfoxide-controlled SN2 displacements between cyanocuprates and epoxy vinyl sulfoxides. Tetrahedron Lett. 1996;37(44):8031-8034.

55. Posner GH, Mallamo JP, Miura K. High asymmetric induction during organometallic .beta.-addition to .alpha.,.beta.-ethylenic sulfoxides Synthesis of optically active. beta.-alkylcarboxylic acids, .beta.-substituted cyclopentanones, and steroidal 11-oxoequilenin methyl ether. J Am Chem Soc. 1981;103(10):2886-2888.

56. Abbot DJ, Colonna S, Stirling CJM. Asymmetric induction in nucleophilic addition to an $\alpha \beta$-unsaturated sulphoxide. J Chem Soc D: Chem Commun. 1971;(10):471-471.

57. Pyne SG. Intramolecular addition of amines to chiral vinyl sulfoxides, total synthesis of (R)-(+)-canadine. Tetrahedron Lett. 1987;28(40):4737-4740.

58. Pyne SG, Chapman, SL. Asymmetric intramolecular conjugate addition of amines to chiral vinyl sulphoxides. Total synthesis of (R)-(+)-carnegine. $J$ Chem Soc Chem Commun.1986;(23):1688-1689.

59. Solladie G, Moine G. J Am Chem Soc. 1984;104:6097.

60. Alayrac C, Fromont C, Metzner P, et al. Angew Chem Int Ed Engl. 1997;36:371.

61. Metzner P, Alayrac C, Julienne K, et al. Actualité Chimique. 2000;54.

62. Koichi N, Yuh-ichiro I, Hideki K. Stereoselective Preparation of $\beta$-CGlycosides from 2-Deoxyribose Utilizing Neighboring Participation by 3-O-Methylsulfinylethyl Group. Chem Lett. 1987;16(11):2139-2142.

63. Montanari F, Danieli R, Hogeveen H. Neighbouring group participation by sulphinyl-oxygen. Tetrahedron Lett. 1964;5(38):2685-2689.

64. Andrew DW, Mark TP, Christopher MR. Homochiral 2,3-epoxy sulfidespowerful new synthetic building blocks providing stereoselective access to 2,3-epoxy sulfoxides, 2,3-dihydroxy sulfoxides and (E)- $\gamma$-hydroxy- $\alpha, \beta$ unsaturated sulfoxides and sulfones. X-Ray molecular structure of rac(2R*,3R*)-1-[(S*)-phenylsulfinyl] hexane-2,3-diol. J Chem Soc Perkin trans I. 1995;(7):847-859.

65. Horner VL, Binder V. Liebig Ann Chem. 1972;33:757.

66. David RD, Ved PD, Daniel CJ. Bromohydrin formation in dimethyl sulfoxide. J Am Chem Soc. 1968;90(20):5498-5501.

67. Sadagopan Raghavan, Ravikumar Ch, Ganapathy Subramanian S. Stereoselective Formal Synthesis of (+)- and (-)-Cyclophellitol and $(-)$-Conduritol-B and Synthesis of $(-)$-Conduramine-B Derivative Using a Sulfinyl Moiety for $\mathrm{C}-\mathrm{O}$ Bond Formation and $\alpha$-Chloro Sulfide for $\mathrm{C}-\mathrm{C}$ Bond Formation. J Org Chem. 2016;81(10):4252-4261.

68. Sadagopan R, Ganapathy Subramanian S. Toward a modular, bidirectional synthesis of (-)-mucocin. Tetrahedron. 2011;67(39):7529-7539.

69. Sadagoapan Raghavan, Ganapathy Subramanian S, Tony KA. A formal convergent synthesis of $(+)$-trans-solamin. Tetrahedron Lett. 2008;49(10):1601-1604. 